\title{
Channel Estimation and Compensation in Chromatic Dispersion Limited Optical Fast OFDM Systems
}

\author{
Jian Zhao and Andrew D. Ellis \\ Tyndall National Institute/Department of Physics, University College Cork, Lee Malting, Cork, Ireland \\ jian.zhao@tyndall.ie, andrew.ellis@tyndall.ie
}

\begin{abstract}
We experimentally investigate the channel estimation and compensation in a chromatic dispersion (CD) limited 20Gbit/s optical fast orthogonal frequency division multiplexing (F-OFDM) system with up to $840 \mathrm{~km}$ transmission. It is shown that symmetric extension based guard interval (GI) is required to enable CD compensation using one-tap equalizers. As few as one optical F-OFDM symbol with four and six pilot tones per symbol can achieve near-optimal channel estimation and compensation performance for $600 \mathrm{~km}$ and $840 \mathrm{~km}$ respectively.
\end{abstract}

\section{INTRODUCTION}

Optical fast orthogonal frequency division multiplexing (F-OFDM) [1-5], with sub-channel spacing equal to half of that in conventional OFDM, is a promising multi-carrier scheme. The sub-carrier multiplexing/demultiplexing can be implemented by using the inverse discrete cosine transform (IDCT)/DCT. The excellent energy concentration property of DCT results in the enhanced robustness to frequency offset [6] and improved performance in channel estimation [7] when compared to conventional OFDM [8, 9]. Furthermore, the DCT, which has been widely adopted in image standards, uses only real arithmetic in contrast to the discrete Fourier transform (DFT) in conventional optical OFDM, whose output is complex even when the input is real. This not only reduces the implementation cost for cost-sensitive applications, but also increases resilience to in-phase/quadrature imbalance.

Due to the different properties of DFT and DCT, the techniques of conventional OFDM cannot be simply employed for F-OFDM and it is necessary to implement solutions specific to optical F-OFDM. In particular, the diagonalizing property of DFT for a circulant channel matrix is not applicable to DCT, such that cyclic prefix (CP) based guard interval (GI) cannot enable ideal chromatic dispersion (CD) compensation using one-tap equalizers. One solution is to use frequency or time domain equalization before channel demultiplexing [3-4], which however increases the implementation complexity. Extending the whole data sequence can enable CD compensation using one-tap equalizers [10-11], but reduces the information throughput by a factor of two. In wireless, other methods such as zero padding [6] or frontend pre-filtering [12] have been used to mitigate fading effects. In [5], it has been shown that the CD-induced channel matrix can be diagonalized by DCT when symmetric extension (SE) based GI is used, enabling CD to be compensated using one-tap equalizers after DCT without any pre-filtering. In this paper, we will experimentally characterize the channel estimation and compensation in a CD-limited 20Gbit/s optical F-OFDM system based on double-side band four-level amplitude shifted keying (4-ASK) sub-carrier modulation with transmission up to $840 \mathrm{~km}$. It is confirmed that SE-based rather than $\mathrm{CP}$-based $\mathrm{GI}$ is required to enable $\mathrm{CD}$ compensation using one-tap equalizers. As few as one FOFDM symbol with six pilot tones per symbol can achieve near-optimal channel estimation and compensation performance for $840 \mathrm{~km}$ transmission.

\section{EXPERIMENTAL SETUP}

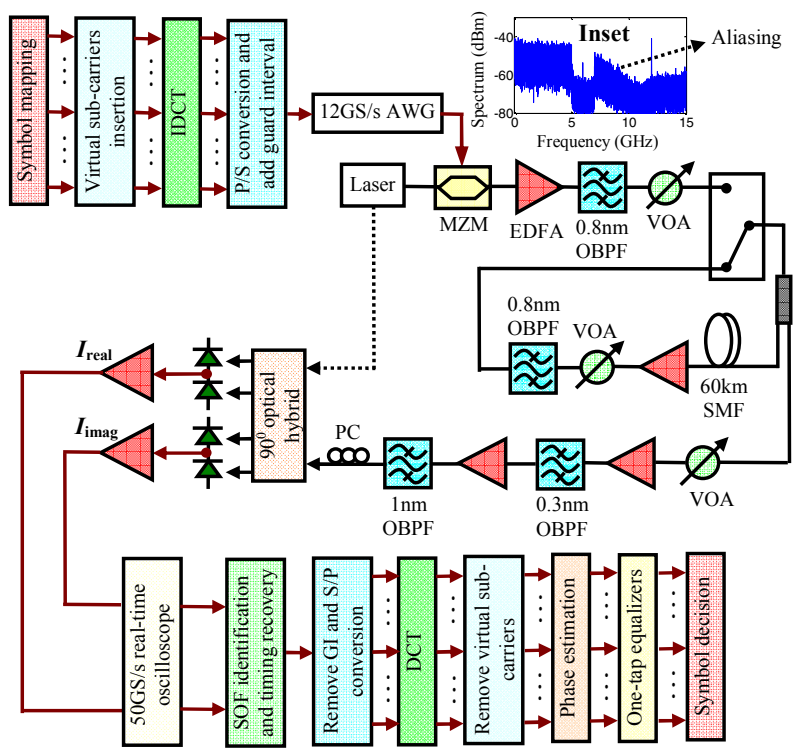

Fig. 1. Experimental setup of 20Gbit/s 4-ASK optical F-OFDM.

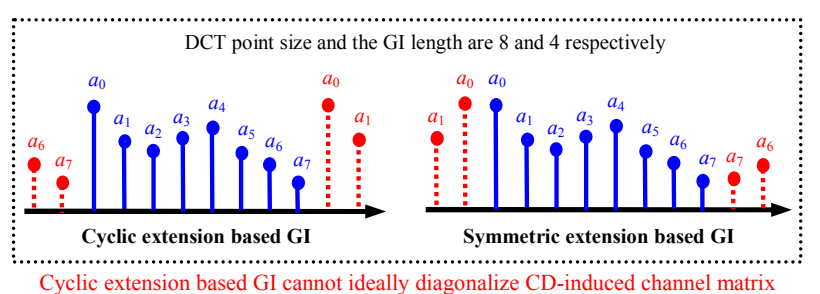

Fig. 2. Cyclic and symmetric extension based guard interval. 
Fig. 1 shows the experimental setup. The bi-polar 4ASK based F-OFDM signal was encoded with gray code in Matlab. The inverse-DCT (IDCT) and DCT used 256 points, of which 213 sub-carriers (sub-carriers \#2-\#214) were used for data transmission. The first sub-carrier (DC) was not modulated, allowing for AC-coupled driving amplifiers and receivers. After IDCT and parallelto-serial $(\mathrm{P} / \mathrm{S})$ conversion, $0,2,6$, or 12 samples were added to each symbol as a SE based GI, whose principle is depicted in Fig. 2. By using the SE-based GI, the CDinduced channel matrix could be represented as the sum of a symmetric Toeplitz matrix and a Hankel matrix [5], which could be diagonalized by DCT/IDCT [12]. Consequently, at the receiver, each F-OFDM symbol could be demultiplexed by DCT without inter-carrier interference and $\mathrm{CD}$ only resulted in different constants multiplied to different sub-carrier data. For comparison, CP based GI with 12 samples was also investigated.

The generated F-OFDM signal was downloaded to a $12 \mathrm{GS} / \mathrm{s}$ arbitrary waveform generator (AWG) with a resolution of 8 bits. The nominal signal line rate including the GI, forward error correction overhead, etc was $10 \mathrm{Gsym} / \mathrm{s}(12 \times 213 / 256)$. The inset of Fig. 1 depicts the electrical spectrum after the AWG, where the electrical bandwidth for 20Gbit/s 4-ASK F-OFDM signal was $5 \mathrm{GHz}$ due to the reduced sub-channel spacing equal to half of the symbol rate per sub-carrier. The frequency components beyond $7 \mathrm{GHz}$ were due to aliasing. A fibre laser with $6 \mathrm{kHz}$ linewidth was used to generate the optical carrier. A Mach-Zehnder modulator (MZM) was used for signal modulation with a peak-to-peak signal input voltage of around $0.5 V_{\pi}$. The modulated optical signal was then amplified by an erbium doped fibre amplifier (EDFA), filtered by a $0.8 \mathrm{~nm}$ optical band-pass filter (OBPF), and transmitted over a recirculating loop comprising $60 \mathrm{~km}$ single-mode fibre (SMF) with $13 \mathrm{~dB}$ fibre loss. The noise figure of the loop EDFA was $5 \mathrm{~dB}$ and another $0.8 \mathrm{~nm}$ OBPF was used in the loop to suppress the amplified spontaneous emission noise. The launch power was set to be $-4.5 \mathrm{dBm}$ using a variable optical attenuator (VOA).

At the receiver, the optical signal was detected with a pre-amplified coherent receiver and a VOA was used to vary the optical signal-to-noise ratio (OSNR). The preamplifier was followed by an OBPF with a $3 \mathrm{~dB}$ bandwidth of $0.3 \mathrm{~nm}$, a second EDFA, and another optical filter with a $3 \mathrm{~dB}$ bandwidth of $1 \mathrm{~nm}$. A polarization controller (PC) was used to align the polarization of the filtered F-OFDM signal before entering the signal path of a $90^{\circ}$ optical hybrid. A tap of the transmitter laser signal was used as the local oscillator at the receiver. The optical outputs of the hybrid were connected to two balanced photodiodes with $40 \mathrm{GHz} 3 \mathrm{~dB}$ bandwidths, amplified by $40 \mathrm{GHz}$ electrical amplifiers, and captured using a 50GS/s real-time oscilloscope. The received samples were interpolated and down sampled to $12 \mathrm{GS} / \mathrm{s}$. An automatic symbol synchronization algorithm as described in [4] was used to not only identify the start-offrame (SOF) symbol but also precisely determine the correct timing position for the DCT window. Then the samples were serial-to-parallel ( $\mathrm{S} / \mathrm{P})$ converted. By using SE-based GI, the sub-carrier data could be firstly demultiplexed by DCT, followed by phase estimation and $\mathrm{CD}$ compensation using one-tap equalizers. The coefficients of the one-tap equalizers were estimated using two methods: 1) all data sub-carriers as pilot tones in training symbols; 2) a few sub-carriers as pilot tones in F-OFDM symbols with subsequent cubic-based frequency-domain interpolation. Eight hundred (800) FOFDM symbols were measured, giving a total number of measured 4 -ASK data of $213 \times 800=170,400$. The bit error rate (BER) was obtained using direct error counting with optimal decision thresholds for each sub-carrier data.

\section{RESULTS AND DISCUSSIONS}

Fig. 3 depicts the performance of 4-ASK optical FOFDM using SE-based GI (solid symbols) after $600 \mathrm{~km}$ and $840 \mathrm{~km}$, in comparison with CP-based GI (empty symbols). It is shown that when CP-based GI is used, the performance is significantly degraded and BER of $10^{-3}$ cannot be achieved even using a GI length of 12 at $\sim 17 \mathrm{~dB}$ OSNR. In contrast, SE-based GI results in better performance, and the required OSNRs to achieve BER of $10^{-3}$ for $600 \mathrm{~km}$ and $840 \mathrm{~km}$ are around $13 \mathrm{~dB}$. The slight penalty for the $840 \mathrm{~km}$ case with respect to the $600 \mathrm{~km}$ case may have been caused by fibre nonlinearity [5], the effect of cascaded filtering in the loop, and depolarization during transmission.

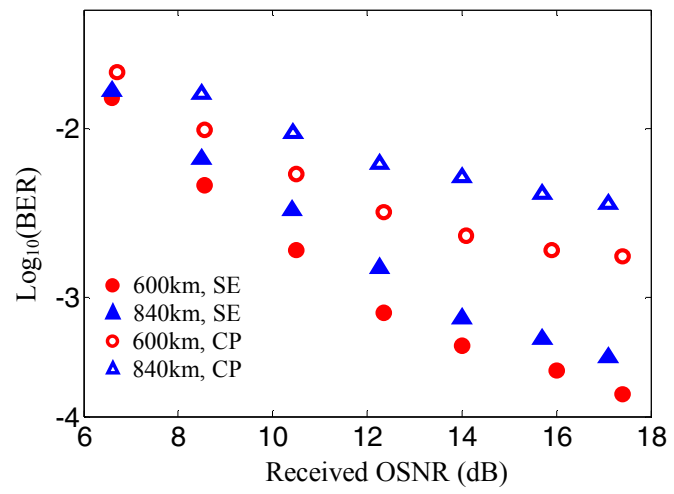

Fig. 3. Performance versus received OSNR for $600 \mathrm{~km}$ (circles) and $840 \mathrm{~km}$ (triangles) using SE-based (solid symbols) and CP-based (empty symbols) GI with length of 12. Channel estimation is obtained using all data sub-carriers as pilot tones in 20 training symbols.

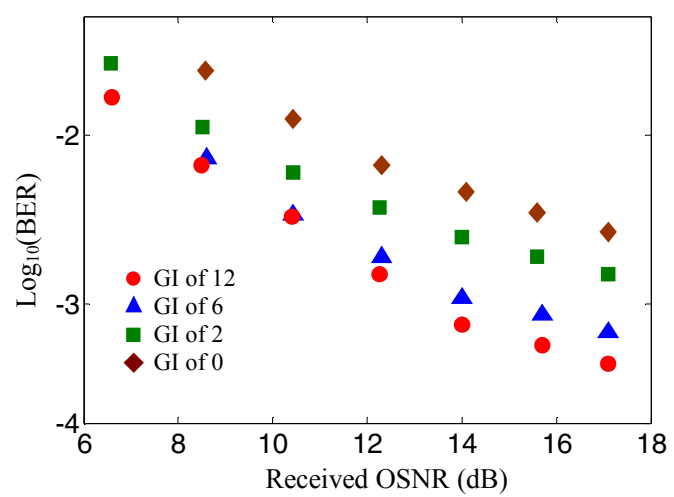

Fig. 4. Performance versus received OSNR for different SE-based GI lengths at $840 \mathrm{~km}$. Channel estimation is obtained using all data subcarriers as pilot tones in 20 training symbols. 


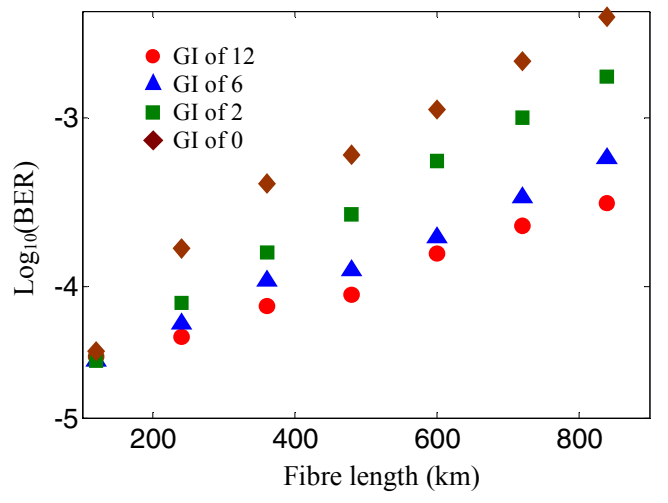

Fig. 5. Performance versus fibre length at $-34 \mathrm{dBm}$ received power (OSNR: 17.1 17.9dB). Channel estimation is obtained using all data sub-carriers as pilot tones in 20 training symbols

To characterize the relation between the transmission performance and the GI length, Fig. 4 shows BER versus received OSNR for different SE-based GI lengths at $840 \mathrm{~km}$. It is straightforwardly observed that a longer GI length results in better performance. This conclusion is further confirmed by Fig. 5, where BER versus fibre length for different GI lengths at $-34 \mathrm{dBm}$ is depicted. Note that at $-34 \mathrm{dBm}$ received power, the OSNR values are different at different fibre lengths, varying from $17.9 \mathrm{~dB}$ for $120 \mathrm{~km}$ to $17.1 \mathrm{~dB}$ for $840 \mathrm{~km}$. When compared to a GI length of 12 , the case without GI results in degraded performance even at $240 \mathrm{~km}$. The use of 2-sample GI length ensures small penalties up to $360 \mathrm{~km}$, after which, however, the BER is degraded severely. Further increasing the GI length to 6 can obtain similar performance as that for the GI length of 12 for up to $600 \mathrm{~km}$ transmission. The use of a GI length longer than 12 might slightly improve the performance, but would reduce the net transmission rate, and an appropriate balance is required in practice.

Previous results are based on the use of all data subcarriers as pilot tones and 20 training symbols. In practice, it is desirable to use as few as pilot tones per F-OFDM symbol and minimized training symbols. This is of particular value for future packet switched optical networks and applications where the $C D$ values may change frequently such that pilot tones are inserted in the payload symbols to track channel changes instantly. We investigate the system performance as a function of the number of pilot tones, as shown in Fig. 6. 20 symbols are used for training and the number of pilot tones is varied. Similar to the GI length, the required number of pilot tones increases with the transmission distance. The figure shows that four and six pilot tones can achieve nearoptimal performance for $600 \mathrm{~km}$ and $840 \mathrm{~km}$ respectively. Fig. 7 shows BER versus the number of training symbols for the two channel estimation methods as described in Section II. The figure shows that using six sub-carriers as pilot tones with subsequent frequency-domain interpolation, whilst significantly reducing the overhead, could achieve similar performance as full channel estimation for large training symbol numbers. Furthermore, this method exhibits negligible and moderate penalties for $600 \mathrm{~km}$ and $840 \mathrm{~km}$ respectively even when only one symbol is used for training. This makes optical F-OFDM a very attractive solution for future packet switched optical networks with fast-varying $C D$ values.

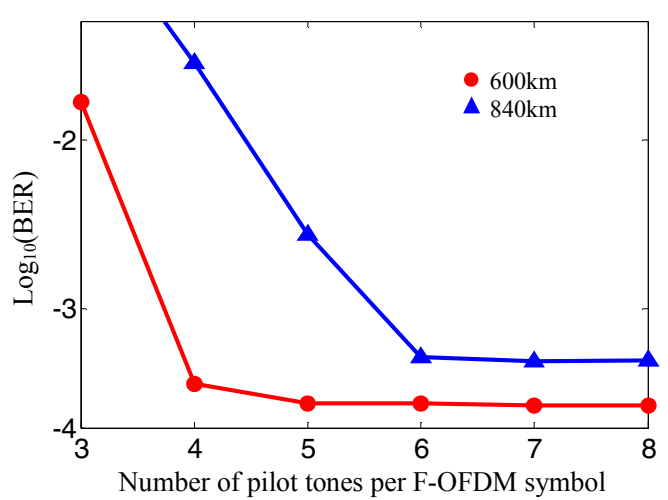

Fig. 6. Performance versus the number of pilot tones per F-OFDM symbol at $-34 \mathrm{dBm}$ received power (OSNR: $17.1 \mathrm{~dB}$ and $17.4 \mathrm{~dB}$ for $840 \mathrm{~km}$ and $600 \mathrm{~km}$ respectively). The SE-based GI length is 12 and the number of training symbols is 20 .

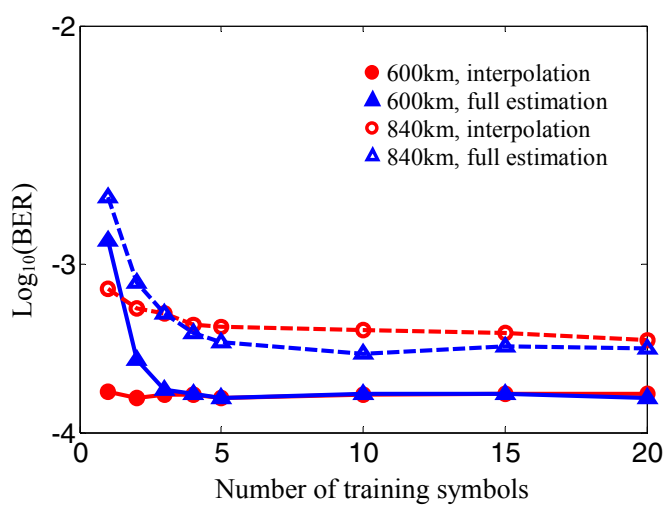

Fig. 7. Performance versus the number of training symbols at $-34 \mathrm{dBm}$ received power (OSNR: $17.1 \mathrm{~dB}$ and $17.4 \mathrm{~dB}$ for $840 \mathrm{~km}$ and $600 \mathrm{~km}$ respectively) for two channel estimation methods. The SE-based GI length is 12 . For circles, the number of pilot tones per symbol is 6 .

\section{CONCLUSIONS}

We have investigated the channel estimation and compensation in a CD-limited 20Gbit/s 4-ASK optical FOFDM system. It is shown that SE, rather than CP, based GI is required to enable $\mathrm{CD}$ compensation using one-tap equalizers. As few as one optical F-OFDM symbol with four and six pilot tones per symbol can achieve nearoptimal performance for $600 \mathrm{~km}$ and $840 \mathrm{~km}$ respectively. This makes optical F-OFDM a very attractive solution for future packet switched optical networks with fast-varying $\mathrm{CD}$ values.

\section{ACKNOWLEDGMENT}

This work was supported by Science Foundation Ireland under grant number 06/IN/I969 and 11/SIRG/I2124.

\section{REFERENCES}

[1] J. Zhao and A.D. Ellis, "A novel optical fast OFDM with reduced channel spacing equal to half of the symbol rate per carrier," Proceedings of Optical Fiber Communication Conference (OFC), paper OMR1, San Diego, CA, 2010.

[2] J. Zhao and A.D. Ellis, "Discrete-Fourier transform based implementation for optical fast OFDM," Proceedings of European Conference on Optical Communication (ECOC), paper Tu.4.A.3, Torino, Italy, 2010. 
[3] S.K. Ibrahim, J. Zhao, D. Rafique, J. O'Dowd, and A.D. Ellis "Demonstration of world-first experimental optical fast OFDM system at $7.174 \mathrm{Gbit} / \mathrm{s}$ and $14.348 \mathrm{Gbit} / \mathrm{s}, "$ Proceedings of European Conference on Optical Communication (ECOC), postdeadline paper PDP3.4, Torino, Italy, 2010.

[4] J. Zhao, S.K. Ibrahim, D. Rafique, P. Gunning, and A.D. Ellis, "Symbol synchronization exploiting the symmetric property in optical fast OFDM,” IEEE Photon. Technol. Lett., vol. 23, pp. 594-596, 2011.

[5] J. Zhao and A.D. Ellis, "Transmission of 4-ASK optical fast OFDM with chromatic dispersion compensation," IEEE Photon. Technol. Lett., vol. 24, pp. 34-36, 2012.

[6] P. Tan and N.C. Beaulieu, "A comparison of DCT-based OFDM and DFT-based OFDM in frequency offset and fading channels," IEEE Trans. Commun., vol. 54, pp. 2113-2125, 2006.

[7] Y. Yeh and S. Chen, "Efficient channel estimation based on discrete cosine transform," Int. Conf. Acoust. Speech Signal Process, pp. 676-679, Hong Kong, 2003.

[8] S.L. Jansen, I. Morita, T.C.W. Schenk, N. Takeda, and H. Tanaka, "Coherent optical $25.8 \mathrm{~Gb} / \mathrm{s}$ OFDM transmission over $4160 \mathrm{~km}$ SSMF," IEEE Journal of Lightwave Technology, vol. 26, pp. 6-15, 2008.

[9] M. Nolle, L. Molle, D.D. Gross, and R. Freund, "Transmission of $5 \times 62 \mathrm{Gbit} / \mathrm{s}$ DWDM coherent OFDM with a spectral efficiency of $7.2 \mathrm{bit} / \mathrm{s} / \mathrm{Hz}$ using joint 64QAM and 16QAM modulation," Proceedings of Optical Fiber Communication Conference (OFC), paper OMR4, San Diego, CA, 2010.

[10] G. Mandyam, "On the discrete cosine transform and OFDM systems," Int. Conf. Acoust. Speech Signal Process, pp. 544-547, Hong Kong, 2003.

[11] E. Giacoumidis, S. K. Ibrahim, J. Zhao, J. M. Tang, A. D. Ellis, and I. Tomkos, "Experimental and Theoretical Investigations of Intensity-Modulation and Direct-Detection Optical Fast-OFDM over MMF-links," IEEE Photon. Technol. Lett., vol. 24, pp. 52-54, 2012

[12] N.A. Dhahir and H. Minn, "A new multicarrier transceiver based on the discrete cosine transform," IEEE Wireless Communication and Networking Conference, pp. 45-50, New Orleans, LA, 2005. 